\title{
Activation of the unfolded protein response in aged human lenses
}

\author{
HE-ZHEN TANG and LI-MIN YANG \\ Department of Ophthalmology, Liaocheng People's Hospital, Liaocheng, Shandong 252000, P.R. China
}

Received March 15, 2014; Accepted November 20, 2014

DOI: $10.3892 / \mathrm{mmr} .2015 .3417$

\begin{abstract}
Cataract formation is a multifactorial disease, induced by a variety of stressors. The endoplasmic reticulum (ER) stress-induced unfolded protein response (UPR) is known to produce reactive oxygen species (ROS) leading to apoptosis. The present study aimed to investigate whether activation of the UPR occurs in human lenses, using human lens epithelial cell (HLEC) lines and lenses obtained from an eye bank, from individuals aged between 50 and 90 years. In vitro analysis was performed using calcimycin $(10 \mu \mathrm{M})$ as an ER stressor. The level of ER stress was measured by the production of ROS, staining for cell death, detection of binding immunoglobulin proteins (BIP) and levels of other UPR proteins, including inositol-requiring enzyme-1 (IRE), activating transcription factor (ATF) 6 and PKR-like eukaryotic initiation factor 2a kinase (PERK). These parameters were examined in HLECs exposed to calcimycin for 12, 24, 48 and $72 \mathrm{~h}$. Fluorescent activated cell sorting analysis of the levels of ROS and apoptosis revealed an increase following $24 \mathrm{~h}$ calcimycin exposure. The reverse transcription quantitative polymerase chain reaction results demonstrated a gradual increase in the mRNA levels of BIP, IRE1, ATF6 and PERK between 12 and $72 \mathrm{~h}$. A similar effect was observed in the protein levels, which also demonstrated a gradual increase in the levels of endoplasmic oxidoreductin-1-like (Ero1-L)- $\beta$ and protein disulfide isomerase, but a lower level of Ero1-L $\alpha$. Activation of the UPR involved the apoptotic pathway, revealed by increased levels of C/EBP homologous protein, ATF4 and caspase-4. Additionally, the antioxidant protein levels were also suppressed. The investigation of aged human lenses revealed a similar increase in the protein expression of UPR. These results indicated that activation of the UPR-induced ROS production suppressed the antioxidant status and triggered the apoptotic pathway, ultimately leading to the formation of age-related cataracts.
\end{abstract}

Correspondence to: Dr Li-Min Yang, Department of Ophthalmology, Liaocheng People's Hospital, 67 Dongchang Road, Liaocheng, Shandong 252000, P.R. China

E-mail: yanglimin20@gmail.com

Key words: age-related cataract, unfolded protein response, endoplasmic reticulum stress, human lens epithelial cells

\section{Introduction}

The unfolded protein response (UPR) is an adaptive cellular response, which is involved in the attenuation of protein synthesis to prevent distorted protein aggregation and the activation of endoplasmic reticulum (ER)-associated distorted protein degradation to accelerate the elimination of protein aggregates (1-5). Conversely, extended UPR activation causes ER stress, which leads to apoptosis and activation of activating transcription factor (ATF) (6). ATF6 activates ATF4, a central transcriptional activator, which activates the apoptotic factor, C/EBP homologous protein (CHOP) (6). The UPR also activates caspase-12 (7-9) and disrupts the $\mathrm{Ca}^{2+}$ balance in the ER (10-12). The UPR apoptotic pathway generates reactive oxygen species (ROS), which are derived from the UPR-associated oxidative protein folding machinery in the ER through protein disulfide isomerase (PDI), coenzyme endoplasmic oxidoreductin-1-like (Ero1-L) and decreased levels of glutathione (13-15).

Previous studies have revealed that the UPR is associated with aging and potentially UPR-induced age-related disease, including cataracts (16-18). Age-related cataracts (ARCs), a major type of cataract, are considered to occur as a result of normal aging processes. Aging, combined with environmental and genetic stresses, is considered to be the predominant contributor to the pathogenesis of lens oxidation, crystallin modification and aggregation (19). However, several of the pathogenic mechanisms involved in ARC remain to be elucidated. Oxidative stress is considered to be one of the cellular conditions associated with the development of ARC $(20,21)$. This is induced by a range of factors, including diabetes, malnutrition, systemic and ocular disease processes, pollutants, drugs, heavy metals, ionizing radiation, glucose and changes in the oxygenation of cells $(20,21)$. These stress factors result in the generation of $\operatorname{ROS}(16,22,23)$.

Based on emerging evidence of UPR activation and its association with aging, the present study aimed to investigated whether UPR activation occurs in aging human lens cells, which may be involved in the formation of ARCs.

\section{Materials and methods}

Age-related human lenses and cell culture. Human lenses aged between 50 and 90 years were obtained from The Eye Bank of Shandong Eye Institute (Qingdao, China) for the detection of UPR proteins in the lens. The human lens epithelial cell (HLEC) line was used for in vitro experiments. HLECs 
were previously stored in liquid nitrogen and then maintained in $25 \mathrm{mM}$ glucose Dulbecco's modified Eagle's medium (DMEM; Invitrogen Life Technologies, Carlsbad, CA, USA) with $10 \%$ fetal calf serum at $37^{\circ} \mathrm{C}$ in $5 \%$ atmospheric oxygen. The HLECs were pre-cultured overnight in $5 \mathrm{mM}$ glucose DMEM in a $\mathrm{CO}_{2}$ incubator (Thermo Fisher Scientific, Inc., Waltham, MA, USA) for use in the subsequent experiments.

ROS and apoptosis measurements. The HLECs were cultured for $12,24,48$ and $72 \mathrm{~h}$ prior to harvesting to determine the levels of ROS and apoptosis. The cells $\left(\sim 5 \times 10^{6}\right)$ were collected by centrifugation and subsequently resuspended in $250 \mu \mathrm{l}$ phosphate-buffered saline (PBS; $\mathrm{pH} 7.4$ ), containing $20 \mu \mathrm{M}$ dihydrorhodamine. After $30 \mathrm{~min}$ incubation at room temperature in the dark, the cells were washed using PBS and resuspended in $500 \mu \mathrm{l}$ PBS for fluorescence activated cell sorting (FACS) analysis using a BD FACS Aria ${ }^{\mathrm{TM}}$ II (BD Biosciences, Franklin Lakes, NJ, USA). The measure the levels of apoptosis, the cells were counterstained with propidium iodide $(2 \mu \mathrm{g} / \mathrm{ml})$ at $4^{\circ} \mathrm{C}$. The cells were filtered through a $50 \mu \mathrm{m}$ nylon mesh (BD Biosciences) to remove cell clumps and were seeded into FACS tubes for FACS analysis to assess levels of apoptosis.

Reverse transcription-quantitative polymerase chain reaction (RT-qPCR). The total RNA was extracted from the HLECs, following treatment with or without varying concentrations of homocysteine using a Quick-RNA ${ }^{\mathrm{TM}}$ MicroPrep solution (Zymo Research Corporation, Orange, CA, USA) according to the manufacturer's instructions. Subsequently, the purified total RNA was reverse transcribed using iScript ${ }^{\mathrm{TM}}$ Reverse Transcription supermix (Bio-Rad Laboratories, Inc., Hercules, CA, USA) according to the manufacturer's instructions. The reverse transcribed RNA was analyzed by RT-qPCR using a SsoFast $^{\mathrm{TM}}$ EvaGreen supermix (Bio-Rad Laboratories, Inc.). The primer sequences were designed for optimal design using OligoPerfect ${ }^{\mathrm{TM}}$ Designer software (Invitrogen Life Technologies), according to the manufacturer's instructions and were synthesized commercially (Invitrogen Life Technologies). The primer sequences used were as follows: Binding immunoglobulin protein (BIP), forward (5'-GGAAAGAAGGTTACCC ATGC-3') and reverse (5'-TTAGGCCAGCAATAGTTCCA-3'); inositol-requiring enzyme-1 (IRE1), forward (5'-AGACTTTG TCATCGGCCTTT-3') and reverse (5'-TGTATTCTGTTCGC CCAAGA-3'); ATF6 forward (5'-GATTAAAGGCTGCCCTC TCA-3') and reverse (5'-GCCTCTGGTTCTCTGACACA-3'); PKR-like eukaryotic initiation factor 2a kinase (PERK), forward (5'-CACCAGAGAAGTGGCAAGAA-3') and reverse (5'-CATCCATTGGGCTAGGAGAG-3') and $\beta$-actin forward (5'-CCAACCGCGAGAAGATGA-3') and reverse (5'-CCAGA GGCGTACAGGGATAG-3'). The PCR conditions included: $2 \mathrm{~min}$ at $95^{\circ} \mathrm{C}, 20 \mathrm{sec}$ at $95^{\circ} \mathrm{C}, 20 \mathrm{sec}$ at the annealing temperature, $30 \mathrm{sec}$ at $72^{\circ} \mathrm{C}$ for 35 cycles and $10 \mathrm{~min}$ at $72^{\circ} \mathrm{C}$. Each reaction was performed in triplicate in three independent experiments. A standard curve (18S rRNA) was constructed from a dilution series of a reference cDNA sample and was included in each RT-qPCR run to correct for possible variations in product amplification. The relative copy numbers were obtained using the standard curve and were normalized to the values obtained for $\beta$-actin, the internal control. The fold change in expression levels were obtained using the $2^{-\Delta \Delta \mathrm{CT}}$ method (24).

Western blot analysis. The HLECs were lysed in radioimmunoprecipitation buffer (Cell Signaling Technology, Inc., Danvers, MA, USA). The soluble proteins (10-20 $\mu \mathrm{g} / \mathrm{l})$ were separated using SDS-PAGE and were subsequently blotted onto polyvinylidene fluoride membranes (Bio-Rad Laboratories, Inc.). The membranes were blocked using 5\% non-fat dry milk for $1 \mathrm{~h}$ at room temperature prior to incubation overnight with primary antibodies at $4^{\circ} \mathrm{C}$. The primary antibodies included: Mouse monoclonal antibodies against Bip $(1: 1,000)$ and IRE1 (1:2,000); rabbit polyclonal ATF6 $(1: 2,000)$ and PERK $(1: 3,000$; BD Biosciences); rabbit polyclonal antibodies against ATF4 (1:500) and catalase $(1: 2,000)$; mouse monoclonal antibodies against CHOP (1:1,000), Erol-L $\alpha$ (1:1,000), Erol-L $\beta$ (1:500), PDI $(1: 2,000)$ and glutathione reductase $(1: 2,000)$, superoxide dismutase (SOD; 1:3,000; Santa Cruz Biotechnology, Inc., Santa Cruz, CA, USA) and mouse monoclonal antibodies against GAPDH $(1: 4,000)$ obtained from Novus Biological (Littleton, CO, USA). The membranes were washed and subsequently incubated with secondary antibodies, including horseradish peroxidase (HRP) conjugated goat anti-rabbit IgG $(1: 5,000)$ and goat anti-mouse IgG-HRP (1:5,000; Santa Cruz Biotechnology, Inc.) for $1 \mathrm{~h}$ at room temperature. Luminol reagent $\left(0.1 \mathrm{ml}\right.$ per $\mathrm{cm}^{2}$ of membrane; Thermo Fisher Scientific) was applied to the membranes and the bands were detected using a Bio-rad XRS system (Bio-Rad Laboratories, Inc.). Antibodies against BIP, IRE1, ATF6, PERK (BD Biosciences), ATF4, CHOP, Ero1-L $\alpha$, Ero1-L $\beta$, PDI, glutathione reductase (GR), catalase, and superoxide dismutase (SOD), from Santa Cruz Biotechnology, Inc., (Santa Cruz, CA, USA) and GAPDH from Novus Biological, (Littleton, CO, USA) were used. The intensity of each band was normalized to GAPDH.

Statistical analysis. The data are expressed as the mean \pm standard deviation and the statistical significance was evaluated using Student's t-test with SPSS (version 15.0) software (SPSS, Inc., Chigago, IL, USA). $\mathrm{P}<0.05$ was considered to indicate a statistically significant difference.

\section{Results}

ROS production and apoptosis. Following exposure of the HLECs to calcimycin for $12,24,48$ and $72 \mathrm{~h}$, a gradual increase was observed in the production of ROS, with a significant increase at $48 \mathrm{~h}$. Similarly, apoptosis was observed after $24 \mathrm{~h}$ and a significant increase was observed after $72 \mathrm{~h}$. ROS production and apoptosis were measured using FACS analysis. ROS production was determined by dihydrorhodamine staining and propidium iodide staining was used to measure apoptotic cell population. The percentage of staining with the respective dyes is shown in Fig. 1A.

mRNA expression levels of UPR-associated genes. The mRNA expression levels of UPR-associated genes were measured using RT-qPCR. The mRNA expression level of BIP gradually increased after $24 \mathrm{~h}$ of exposure, with a significant 10-fold increase after $72 \mathrm{~h}$ (Fig. 1B). BIP is considered a marker for UPR activation, which triggers the activation of 
A
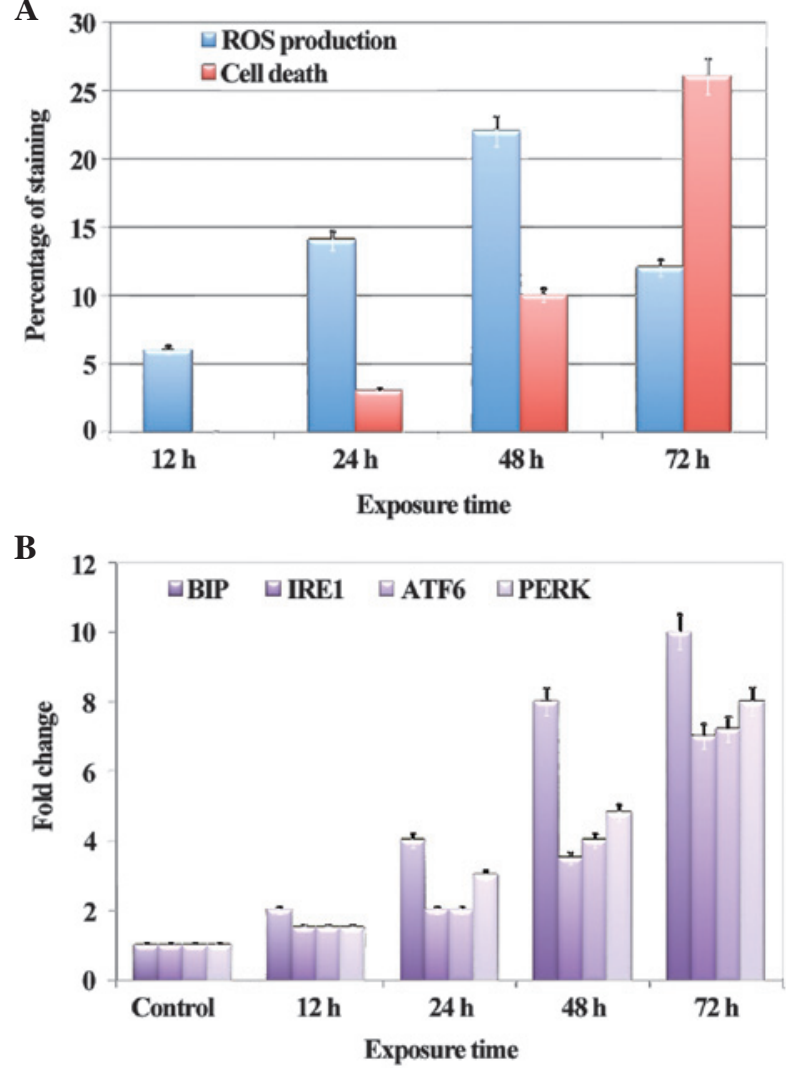

Figure 1. UPR activation in the HLECs. (A) FACS analysis demonstrating the percentage of ROS production and cell death at different time points. (B) Reverse transcription quantitative polymerase chain reaction analysis of the fold change in the mRNA levels of UPR-associated genes. Error bars represent the mean \pm standard error of the mean. UPR, unfolded protein response; HLECs, human lens epithelial cells; FACS, fluorescent activated cell sorting; ROS, reactive oxygen species; BIP, binding immunoglobulin protein; IRE1, inositol-requiring enzyme-1; ATF, activating transcription factor; PERK, PKR-like eukaryotic initiation factor 2a kinase.

other UPR-associated proteins. The mRNA expression levels of UPR-associated genes, including IRE1, ATF6 and PERK, began increasing at $48 \mathrm{~h}$, with a maximum 8 -fold increase at $72 \mathrm{~h}$ (Fig. 1B), indicating the effect of UPR activation in the HLECs following exposure to calcimycin, the ER stressor.

Activation of UPR-associated proteins in the HLECs. The western blot analysis of UPR-associated proteins revealed that the level of BIP gradually increased in the HCLCs after $12 \mathrm{~h}$ of exposure to calcimycin. A significant increase in the protein level of BIP was identified after $24 \mathrm{~h}$ of exposure. The levels of other UPR proteins were elevated after $24 \mathrm{~h}$ of exposure, indicating activation of the UPR in the HLECs (Fig. 2A). As the duration of exposure increased, the levels of the UPR proteins also increased, which was correlated with the activation of the UPR observed in aging human lenses. As a result of UPR activation, the levels of the proteins involved in the protein folding machinery, including PDI and Erol-L $\alpha$, decreased. Conversely, the protein levels of Ero1-L $\beta$, a counter regulator for Ero1-L $\alpha$, increased (Fig. 2B). This confirmed that HLECs exposed to calcimycin led to activation of the UPR.

Suppression of the antioxidant system and activation of apoptosis. The protein levels of antioxidant and apoptotic signaling
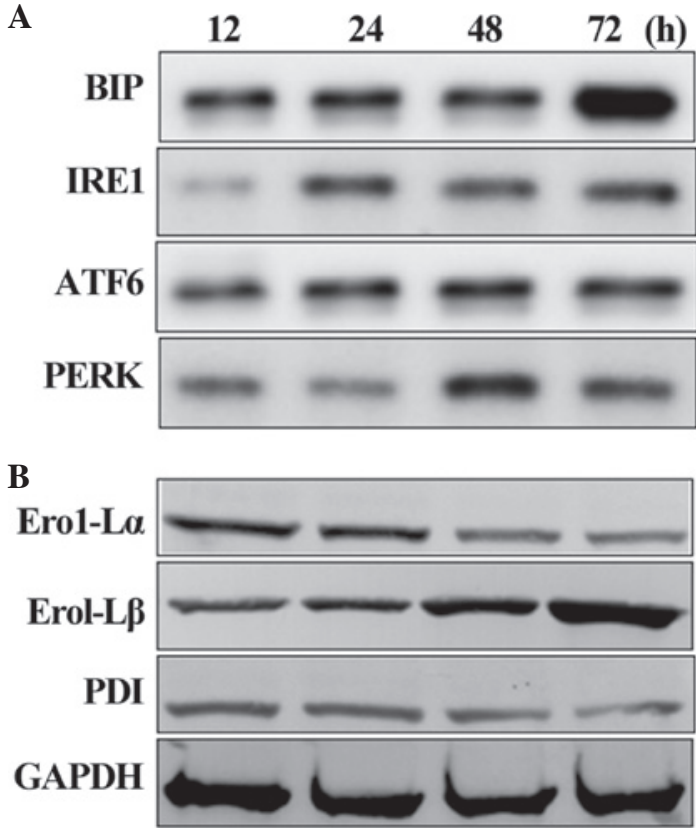

Figure 2. Activation of the UPR in the HLECs. (A) Western blot analysis of the levels of UPR signaling proteins BIP, IRE1, ATF6 and PERK. (B) Western blot analysis of the levels of UPR-associated proteins, which were expressed following UPR activation. UPR, unfolded protein response; HLECs, human lens epithelial cells; ROS, reactive oxygen species; BIP, binding immunoglobulin protein; IRE1, inositol-requiring enzyme-1; ATF, activating transcription factor; PERK, PKR-like eukaryotic initiation factor 2a kinase, Ero1-L, endoplasmic oxidoreductin-1-like; PDI, protein disulfide isomerase.

$\mathbf{A}$
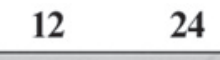

48 72 (h)
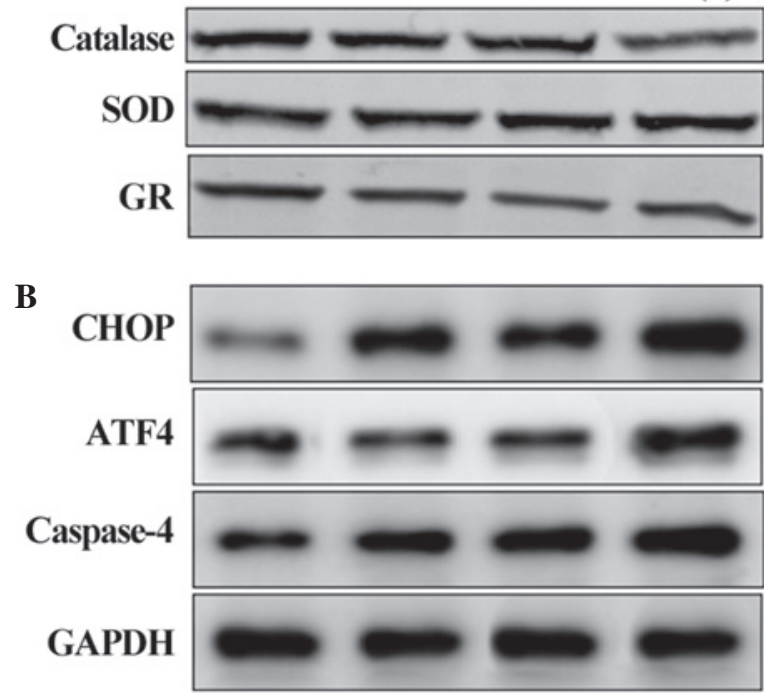

Figure 3. Effect of UPR activation on the (A) antioxidant proteins and (B) signaling proteins involved in the apoptotic pathway. UPR, unfolded protein response; SOD, superoxide dismutase; GR, glutathione reductase; $\mathrm{CHOP}, \mathrm{C} / \mathrm{EBP}$ homologous protein; ATF, activating transcription factor.

proteins were assessed in the HLECs exposed to calcimycin. The protein levels of the antioxidants catalase, SOD and GR decreased after 48 and $72 \mathrm{~h}$ of exposure, indicating that UPR activation suppressed the antioxidant level and produced ROS (Fig. 3A). The levels of the CHOP, ATF4 and caspase-4 apoptotic signaling proteins gradually increased after $24 \mathrm{~h}$ and increased markedly at $72 \mathrm{~h}$ (Fig. 3B). This suggested that the 


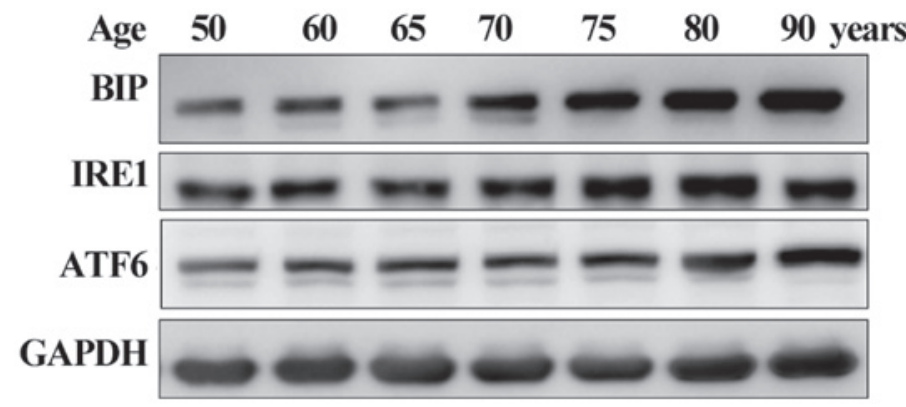

Figure 4. Age-dependent activation of the unfolded protein response in aged human lenses. BIP, binding immunoglobulin protein; IRE1, inositol-requiring enzyme-1; ATF, activating transcription factor.

activation of UPR triggers the ER stress-mediated apoptotic signaling pathway in HLECs.

Age-related activation of the UPR-associated proteins in human lenses. Based on the in vitro results, the present study aimed to confirm whether UPR activation is observed in human lenses aged between 50 and 90 years old. The protein expression of BIP was observed in all the lenses and significantly elevated in lenses aged $\geq 70$ years. The IRE1 and ATF6 UPR proteins gradually increased with age (Fig. 4). These results confirmed that the UPR was activated in the human lenses. Therefore, activation of the UPR was involved in the production of ROS, decreased antioxidant levels and activation of the apoptotic pathway, ultimately leading to lens oxidation or the formation of cataracts.

\section{Discussion}

Cataract formation is a multifactorial disease occurring predominantly in older individuals based on their exposure to internal or external stressors. Cataractogenic factors are responsible for the generation of ROS and the failure of the antioxidant system in the lens epithelial cells, leading to lens oxidation or cataract formation (25). Generally, ROS are generated in the mitochondria of the cell, however, lens epithelial cells lacks mitochondria and the generation of ROS in the lens cells remains to be elucidated $(26,27)$. The present study revealed that ER stress induced the activation of the UPR and that chronic activation of the UPR leads to the generation of ROS and causes apoptosis. At higher levels of calcimycin, misfolded proteins accumulated in the ER, which can then induce the UPR $(28,29)$.

In the present study, HLECs exposed to calcimycin, an ER stressor, for $24 \mathrm{~h}$ led to the formation of ROS and, after $48 \mathrm{~h}$, apoptosis occurred. It was hypothesized that this may be due to activation of the UPR. To investigate this hypothesis, gene expression levels were assessed after 12, 24, 48 and $72 \mathrm{~h}$ of calcimycin exposure. The initial responses of the UPR are the phosphorylation of three ER-associated transmembrane proteins; PERK, IRE1 and ATF6, which are cleaved and separated from the ER membrane (16). These responses lead to translational and transcriptional arrest and a reduction in protein production in order to recover from the accumulation of unfolded proteins in the ER. However, in the presence of continued ER stress, the apoptotic pathway is activated by the central transcriptional activators, ATF4 and ATF6, which subsequently activate the apoptotic factor CHOP (30). Consistent with these results, the present study revealed a significant increase in the mRNA and protein levels of IRE1, ATF6, PERK $72 \mathrm{~h}$ after exposure. The levels of BIP, which is considered to be an ER stress marker, were increased after $24 \mathrm{~h}$. A similar pattern of protein expression levels were identified in the 50-90 year old lenses, in which an age-dependent increase in UPR-associated protein levels was observed. These results demonstrated that UPR activation is common in aging lenses and may trigger the formation of ARC.

To elucidate whether activation of the UPR is involved in altering the antioxidant system to cause apoptosis, the levels of antioxidants and other UPR-associated proteins involved in apoptosis were assessed. The UPR also activates caspase-4 (caspase-12 in rodents) resulting in apoptosis $(29,31)$ and generates excess levels of ROS, driven by PDI, Ero1-L $\alpha$ and Ero1-L $\beta$ (13-15). The production of ROS decreases the levels of cytosolic glutathione and contributes to an additional source of ROS from the mitochondria (32) resulting in apoptosis. The results from the present study were consistent with these findings. The initial step of UPR activation triggered the survival pathway by increasing the levels of PDI and Erol-L $\beta$. Notably, the levels of Erol-L $\alpha$ decreased during the exposure period. The protein levels of catalase, SOD and GR decreased in the cells exposed for $>24 \mathrm{~h}$. Similarly, activation of the UPR induced the apoptotic pathway, which was confirmed by elevated protein levels of ATF4, CHOP and caspase-4.

In conclusion, the present study demonstrated that the UPR was activated in HLECs, which was confirmed in human aged lenses. Furthermore, UPR activation generated excess levels of ROS and altered the antioxidant system. Prolonged exposure to UPR activation triggered apoptotic pathway signaling proteins, leading to lens oxidation or the formation of ARC. Thus, UPR activation is important in the formation of ARC.

\section{References}

1. Kozutsumi Y, Segal M, Normington K, Gething MJ and Sambrook J: The presence of malfolded proteins in the endoplasmic reticulum signals the induction of glucose-regulated proteins. Nature 332: 462-464, 1988.

2. Brewer JW and Diehl JA: PERK mediates cell-cycle exit during the mammalian unfolded protein response. Proc Natl Acad Sci USA 97: 12625-12630, 2000.

3. Prostko CR, Brostrom MA, Malara EM and Brostrom CO: Phosphorylation of eukaryotic initiation factor (eIF) 2 alpha and inhibition of eIF-2B in GH3 pituitary cells by perturbants of early protein processing that induce GRP78. J Biol Chem 267: 16751-16754, 1992. 
4. Shi Y, Vattem KM, Sood R, An J, Liang J, Stramm L, et al: Identification and characterization of pancreatic eukaryotic initiation factor 2 alpha-subunit kinase, PEK, involved in translational control. Mol Cell Biol 18: 7499-7509, 1998.

5. Tirasophon W, Welihinda AA and Kaufman RJ: A stress response pathway from the endoplasmic reticulum to the nucleus requires a novel bifunctional protein kinase/endoribonuclease (Irelp) in mammalian cells. Genes Dev 12: 1812-1824, 1998.

6. Rao RV, Ellerby HM and Bredesen DE: Coupling endoplasmic reticulum stress to the cell death program. Cell Death Differ 11: 372-380, 2004

7. Mandic A, Hansson J, Linder S and Shoshan MC: Cisplatin induces endoplasmic reticulum stress and nucleus-independent apoptotic signaling. J Biol Chem 278: 9100-9106, 2003.

8. Tinhofer I, Anether G, Senfter M, Pfaller K, Bernhard D, Hara M, et al: Stressful death of T-ALL tumor cells after treatment with the anti-tumor agent Tetrocarcin-A. FASEB J 16 : 1295-1297, 2002.

9. Xie Q, Khaoustov VI, Chung CC, Sohn J, Krishnan B, Lewis DE, et al: Effect of tauroursodeoxycholic acid on endoplasmic reticulum stress-induced caspase-12 activation. Hepatology 36: 592-601, 2002.

10. Kaufman RJ, Scheuner D, Schroder M, Shen X, Lee K, Liu CY, et al: The unfolded protein response in nutrient sensing and differentiation. Nat Rev Mol Cell Biol 3: 411-421, 2002.

11. Szegezdi E, Fitzgerald U and Samali A: Caspase-12 and ER stress-mediated apoptosis: the story so far. Ann N Y Acad Sci 1010: 186-194, 2003.

12. Ji C, Deng Q and Kaplowitz N: Role of TNF-alpha in ethanol-induced hyperhomocysteinemia and murine alcoholic liver injury. Hepatology 40: 442-451, 2004.

13. Haynes CM, Titus EA and Cooper AA: Degradation of misfolded proteins prevents ER-derived oxidative stress and cell death. Mol Cell 15: 767-776, 2004

14. McCullough KD, Martindale JL, Klotz LO, Aw TY and Holbrook NJ: Gadd153 sensitizes cells to endoplasmic reticulum stress by down-regulating $\mathrm{Bcl} 2$ and perturbing the cellular redox state. Mol Cell Biol 21: 1249-1259, 2001.

15. Tu BP, Ho-Schleyer SC, Travers KJ and Weissman JS Biochemical basis of oxidative protein folding in the endoplasmic reticulum. Science 290: 1571-1574, 2000.

16. Ikesugi K, Yamamoto R, Mulhern ML and Shinohara T: Role of the unfolded protein response (UPR) in cataract formation. Exp Eye Res 83: 508-516, 2006

17. Elanchezhian R, Palsamy P, Madson CJ, et al: Low glucose under hypoxic conditions induces unfolded protein response and produces reactive oxygen species in lens epithelial cells. Cell Death Dis 3: e301, 2012.
18. Elanchezhian R, Palsamy P, Madson CJ, Lynch DW and Shinohara T: Age-related cataracts: homocysteine coupled endoplasmic reticulum stress and suppression of Nrf2-dependent antioxidant protection. Chem Biol Interact 200: 1-10, 2012.

19. Michael R and Bron AJ: The ageing lens and cataract: a model of normal and pathological ageing, Philos Trans R Soc Lond B Biol Sci 366: 1278-1292, 2011.

20. Harding JJ: Free and protein-bound glutathione in normal and cataractous human lense. Biochem J 117: 957-960, 1970.

21. Lou MF: Redox regulation in the lens. Prog Retin Eye Res 22: 657-682, 2003

22. Ikesugi K, Mulhern ML, Madson CJ, et al: Induction of endoplasmic reticulum stress in retinal pericytes by glucose deprivation. Curr Eye Res 31: 947-953, 2006.

23. Mulhern ML, Madson CJ, Danford A, Ikesugi K, Kador PF and Shinohara T: The unfolded protein response in lens epithelial cells from galactosemic rat lenses. Invest Ophthalmol Vis Sci 47: 3951-3959, 2006.

24. Schmittgen TD and Livak KJ: Analyzing real-time PCR data by the comparative $\mathrm{C}(\mathrm{T})$ method. Nat Protoc 3: 1101-1108, 2008.

25. Shichi H: Cataract formation and prevention. Expert Opin Investig Drugs 13: 691-701, 2004.

26. Bloemendal H, de Jong W, Jaenicke R, Lubsen NH, Slingsby $\mathrm{C}$ and Tardieu A: Ageing and vision: structure, stability and function of lens crystallins. Prog Biophys Mol Biol 86: 407-485, 2004

27. Huang L1, Tang D, Yappert MC, Borchman D: Oxidationinduced changes in human lens epithelial cells 2. Mitochondria and the generation of reactive oxygen species. Free Radic Biol Med 41: 926-936, 2006.

28. Ron D and Walter P: Signal integration in the endoplasmic reticulum unfolded protein response. Nat Rev Mol Cell Biol 8: 519-529, 2007.

29. Schröder M: The unfolded protein response. Mol. Biotechnol 34: 279-290, 2006.

30. Puustjärvi T, Blomster H, KontkanenvM, Punnonen $\mathrm{K}$ and Terasvirta M: Plasma and aqueous humour levels of homocysteine in exfoliation syndrome. Graefes Arch Clin Exp Ophthalmol 242: 749-754, 2004

31. Arap MA, Lahdenranta J, Mintz PJ, Hajitou A, Sarkis AS, Arap W and Pasqualini R: Cell surface expression of the stress response chaperone GRP78 enables tumor targeting by circulating ligands. Cancer Cell 6: 275-284, 2004.

32. Huang HC, Nguyen T and Pickett CB: Phosphorylation of Nrf2 t Ser-40 by protein kinase $\mathrm{C}$ regulates antioxidant response element-mediated transcription. J Biol Chem 277: 42769-42774, 2002. 